\title{
Ultrasound-guided core needle biopsy compared to open biopsy: a new diagnostic approach to major salivary gland enlargement in Sjögren's syndrome
}

Alen Zabotti ( $\nabla$ zabottialen@gmail.com )

Rheumatology clinic, university of Udine, Italy https://orcid.org/0000-0002-0573-464X

Sara Zandonella Callegher

Rheumatology Clinic, Department of Medicine, University of Udine, c/o Azienda sanitaria universitaria

Friuli Centrale, Udine

Michele Lorenzon

Institute of Radiology, ASUFC Udine

\section{Enrico Pegolo}

Institute of Anatomic Pathology, ASUFC, Udine

\section{Cathryn Anne Scott}

Institute of Anatomic Pathology, ASUFC, Udine

Alessandro Tel

Maxillofacial surgery, ASUFC, Udine

Ivan Giovannini

Rheumatology Clinic, ASUFC, Udine

Massimo Robiony

Maxillofacial Surgery, ASUFC, Udine

Carla Di Loreto

Institute of Anatomic Pathology, ASUFC, Udine

Chiara Zuiani

Institute of Radiology, ASUFC, Udine

Salvatore De Vita

Rheumatology Clinic, ASUFC, Udine

Research article

Keywords: Sjögren's syndrome, salivary gland, histopathology, ultrasonography, ultrasound-guided core needle biopsy

Posted Date: May 19th, 2020

DOl: https://doi.org/10.21203/rs.3.rs-28825/v1 
License: (c) (i) This work is licensed under a Creative Commons Attribution 4.0 International License. Read Full License 


\section{Abstract}

Objective Persistent ( $\geq 2$ months) major salivary gland (SG) enlargement in primary Sjögren's syndrome (pSS) patients is a well-known sign of possible involvement by B-cell lymphoma. The study aimed to evaluate the diagnostic accuracy and safety of ultrasound-guided core needle biopsy (US-guided CNB) of major SGs in comparison to open surgical biopsy. Methods Prospective pSS patients (cases) with clinically persistent SG enlargement underwent US-guided CNB, and were compared to retrospective pSS patients (controls) submitted to open surgical biopsy. The features analyzed were pre-biopsy clinical and laboratory findings, biopsy-related complications (reported by the patient with a questionnaire and clinically verified), adequacy of the material for histology and diagnosis rendered. Results Thirteen cases underwent US-guided CNB: in 9/13 biopsy was performed on the parotid and in 4/13 on the submandibular gland. Sufficient material was obtained for pathological diagnosis in all samples. The final diagnoses were: 5/13 (38.5\%) B-cell lymphoma, 1/13 (7.7\%) lympho-epithelial sialadenitis, 4/13 $(30.7 \%)$ other sialadenitis (granulomatous consistent with sarcoidosis, IgG4-related disease, chronic sclerosing, diffuse chronic) and 3/13 (23.1\%) miscellaneous lesions. Thirteen controls underwent open surgical biopsy of the parotid, in one inadequate material was obtained, in $12(92.3 \%)$ the pathologic diagnoses were: 4/12 (33.3\%) B-cell lymphoma, 2/12 (16.7\%) lympho-epithelial sialadenitis, 4/12 (33.3\%) uncertain lymphoproliferative lesions, 2/12 (16.7\%) miscellaneous lesions. 6/13 (46.1\%) cases reported 6 transient complications, and 12/13 (92.3\%) controls 2 persistent and 14 transient complications. Conclusion US-guided CNB represents a novel, clinically relevant and safe approach to the management of pSS patients with parotid or submandibular persistent enlargement.

\section{Introduction}

Primary Sjögren's syndrome (pSS) is an autoimmune and lymphoproliferative connective tissue disease mainly involving the salivary and the lacrimal glands (1). pSS patients are at an increased risk of developing an extranodal marginal zone lymphoma of the major salivary glands (SGs), most frequently of the Mucosa-Associated Lymphoid Tissue Type (MZL-MALT) (2-4). Persistent ( $\geq 2$ months) enlargement of major SGs is one of the main risk factors for B-cell lymphoma in pSS (5-7). It may represent an already established lymphoma or be a manifestation of a pre-lymphomatous process or a different underlying disease $(6,7)$. In this scenario, the biopsy of a persistently swollen major SG is recommended and it is helpful in evaluating the possible subsequent management in the pSS patient. Nevertheless, open surgical biopsy is still not performed routinely due to technical issues (anatomical relation of the facial nerve with the parotid gland), local experience, and development of possible complications such as salivary gland sialocele, fistula, facial nerve injury, etc. (8-10). Ultrasound-guided core needle biopsy (US-guided CNB) is an established, effective and safe procedure that is currently applied for diagnostic purposes also on SGs in relation to epithelial tumors $(11,12)$. US-guided CNB might improve the feasibility of major SGs biopsy also in pSS, a disease in which SGs ultrasonography is increasingly performed for diagnostic and prognostic purposes (13-17). 
This study aimed to: i) evaluate the accuracy and safety of US-guided CNB prospectively in pSS patients with persistent parotid or submandibular gland enlargement; ii) compare US-guided CNB with SGs open surgical biopsy; iii) compare the histopathological diagnosis of the most suspicious sonographic lesion to the adjacent surrounding parenchyma in US-guided CNB cases.

\section{Patients And Methods}

\section{Patients}

Consecutive patients (cases) with either definite pSS according to the American College of Rheumatology/ European League Against Rheumatism (ACR-EULAR) classification criteria (18), or clinically suspected for pSS, referred to the Clinic of Rheumatology, University Hospital of Udine, Italy, from September 2019 to February 2020 were screened for US-guided CNB. The inclusion criteria were: a) presence of unilateral or bilateral parotid and/or submandibular gland enlargement; b) clinical indication for parotid or submandibular biopsy due to the suspicion of a malignant lymphoproliferative disorder, based on the presence of known risk factors for lymphoma $(7,19,20)$. Prior diagnosis of lymphoma was an exclusion criterion, while it was allowed to continue concomitant anticoagulant or antiplatelet therapy.

All patients with definite or suspected pSS (controls) followed in the same center, and who underwent open surgical parotid gland biopsy from February 2016 to June 2019 served as controls. The inclusion criteria were the same as above.

Written informed consent was obtained from each patients in accordance with the Declaration of Helsinki and with local guidelines for good clinical practice. The study was conducted according to a protocol approved by the Regional Ethical Committee (CEUR-2017-Os-027-ASUIUD) (21).

\section{Demographic, Clinical And Laboratory Data}

The following data were collected from the medical charts: gender, date of birth, disease duration, fulfillment of the ACR-EULAR classification criteria for pSS (18), previous minor SGs biopsy, previous positive unstimulated sialometry and Schirmer's test, evidence of serum antinuclear antibodies (ANA), anti-Ro/SSA and anti-La/SSB antibodies. In addition, data relative to known risk factors for lymphoma in pSS were analysed at the time of biopsy: persistent parotid or submandibular gland enlargement, lymphadenopathy, cryoglobulinemic vasculitis or serum cryoglobulinemia, serum monoclonal component, rheumatoid factor, low serum C4, and leukopenia $(7,19,20)$.

\section{Ultrasound-guided Core Needle Biopsy}

US-guided CNBs were performed with an aseptic-freehand biopsy technique under real-time US guidance with linear high-frequency transducers (Samsung RS85, probe LM4-15BMHz or Philips Affiniti 70G, probe 
L18-5) by an extensive expert radiologist (ML) in a non operating room. A 14-gauge semi-automatic core needle biopsy system was used (Precisa 14G, HS Hospital Service); sampling lenght was always set on $20 \mathrm{~mm}$. For parotid biopsies, the patient was in supine position with hyperextension of the neck, and the head slightly turned towards the side opposite to that being biopsied; the entry site was from the posterior glandular border slightly caudal to the ear lobe (Fig. 1-A). For submandibular gland biopsies, the patient was in supine position with hyperextension of the neck and the head turned towards the site being biopsied, an anterior to posterior approach was used. Local infiltration anesthesia was performed through the skin, subcutaneous fat and within the gland and a small skin incision was made with a scalpel. The biopsy samples were sent unfixed for histological analysis. After the procedure, the patients were asked to compress the puncture site and remain under observation for 30 minutes.

The US-guided CNB was performed on the clinically most swollen gland. The choice of gland to be biopsied was clinical, while the site of biopsy was targeted at the most suspicious focal sonographic lesion, when present. One to four needle passes were carried out through the same skin access. A focal glandular lesion was defined as suspicious when either a hypoechoic nodule that displayed sonographic features of a hypertrophic lymphoid structure or a limited area within the SG with hypoechoic lesions larger and/or more confluent than in the remaining parenchyma were present. These sonographic features could be associated with other highly suspicious characteristics, such as lobulated margins, taller than wide appearance and microcalcifications within the lesion (22). In all patients, one or two biopsies of the parenchyma surrounding the focal lesion were carried out through the same skin access.

When the parenchyma was diffusely inhomogeneous, with no focal sonographic lesion, the parotid biopsy was performed in the "safety area", i.e. 1-2 cm beneath the ear lobe, where the facial nerve runs deep beneath the parotid (Fig. 1-A; Additional Figure S1), in order to avoid damage to the facial nerve. The submandibular biopsy was performed in the most superficial glandular parenchyma.

\section{Open Surgical Biopsy}

The open surgical biopsy was performed on the clinically most swollen parotid gland. The parotid gland has a strict anatomical relationship with the facial nerve, so the first step of open surgical biopsy was the correct identification of the so-called "safety zone", i.e., an area localized $1 \mathrm{~cm}$ beneath the ear lobe, which allows to avoid damage of the main trunk of the facial nerve, which is deep in this trait (Fig. 1-B; Additional Figure S1). The procedure was performed under local anesthesia. A small incision was performed, and blunt dissection was carried out until the parotideomasseteric fascia was visualized. The parotid fascia was then incised and the gland parenchyma visualized. A small sample of tissue was harvested and put in a container with formaldehyde. After accurate hemostasis, the parotid fascia was sutured with absorbable sutures, and the overlying skin closed with non-absorbable sutures.

\section{Evaluation Of Post-biopsy Complications}


All patients, regardless of the type of procedure were evaluated clinically between 1 and 2 weeks and 12 weeks following biopsy. Complications that lasted more than 12 weeks were evaluated in scheduled rheumatological evaluations. All patients were asked to complete a questionnaire to report postprocedure complications that were verified by the clinician at follow-up (Additional Figure S2-English version; Additional Figure S3- Italian version). The complications were classified as transient $(<12$ weeks) or persistent ( $\geq 12$ weeks). Any association of the following complications at biopsy site was recorded: swelling, hematoma, bleeding, pain, local infection, anesthesia/paraesthesia, sialocele and fistula.

\section{Histopathological Evaluation}

The material was fixed in $4 \%$ neutral buffered-formalin for 12-24 hours, routinely processed and embedded in paraffin. Four $\mu$ m-thick sections were cut from each block and stained with Haematoxylin \& Eosin. Histo-morphometrical analysis was performed in all cases to calculate the amount of SG tissue in the specimens, using a computer assisted image analysis system (Aperio ImageScope, Leica Biosystems).

The following morphological features were recorded: acinar atrophy, duct dilation, fibrosis, adipose metaplasia, presence of an inflammatory infiltrate with evaluation of cell types (lymphocytes, plasma cells, centrocyte-like lymphocytes), evidence of lymphoid foci, lymphoid follicles with activated germinal centers, and lymphoepithelial lesions (LELs), distribution and tendency to confluence of centrocyte-like lymphocytes (23-25). The immunohistochemical panel consisted of CD3, CD20 and CD21 antibodies, performed to characterize the lymphoid populations and to highlight germinal centers. In some cases, a Pan-Cytokeratin antibody was used to demonstrate the presence of LELs.

Molecular analysis to evaluate B-cell clonality was performed on biopsies showing a lymphoid infiltrate suspicious for lymphoma (IdentiClone ${ }^{\mathrm{TM}}$ IGH Gene Clonality Assay, Invivoscribe).

\section{Results}

\section{Demographic, clinical characteristics of patients and laboratory findings \\ US-guided CNB cases}

Thirteen cases who underwent US-guided CNB were evaluated, 10/13 (76.9\%) fulfilled the ACR-EULAR classification criteria for pSS (18), 12/13 (92.3\%) were female, with a mean age at the time of biopsy of $63.5 \pm 12.1$ years (Table 1; Additional Table S1). Mean disease duration was $11.6 \pm 8.4$ years. Nine out of thirteen $(69.2 \%)$ had a parotid gland enlargement and $4 / 13(30.8 \%)$ a submandibular gland enlargement (Table 1; Additional Table S1). 
Table 1

Patients clinical and laboratory features.

\begin{tabular}{|c|c|c|}
\hline & $\begin{array}{l}\text { US-guided } \\
\text { CNB cases }\end{array}$ & $\begin{array}{l}\text { Open biopsy } \\
\text { controls }\end{array}$ \\
\hline Number of patients & 13 & 13 \\
\hline Gender, female, $n(\%)$ & $\begin{array}{l}12 / 13 \\
(92.3 \%)\end{array}$ & $\begin{array}{l}10 / 13 \\
(76.9 \%)\end{array}$ \\
\hline Age at evaluation, years, mean $\pm S D$ & $63.5 \pm 12.1$ & $51.8 \pm 15.2$ \\
\hline Disease duration, years, mean $\pm S D$ & $11.6 \pm 8.4$ & $8.6 \pm 8.5$ \\
\hline Fulfilment of ACR-EULAR classification criteria for pSS, $n$ (\%) & $\begin{array}{l}10 / 13 \\
(76.9 \%)\end{array}$ & $\begin{array}{l}12 / 13 \\
(92.3 \%)\end{array}$ \\
\hline \multicolumn{3}{|l|}{ Parotid gland enlargement } \\
\hline \multirow{2}{*}{$\begin{array}{l}\text { Chronic ( } \geq 12 \text { months), } n \text { (\%) } \\
\text { Episodically persistent ( } 2-12 \text { months), } n \text { (\%) }\end{array}$} & $6 / 13(46.1 \%)$ & $\begin{array}{l}10 / 13 \\
(76.9 \%)\end{array}$ \\
\hline & $3 / 13(23.1 \%)$ & $3 / 13(23.1 \%)$ \\
\hline \multicolumn{3}{|l|}{ Submandibular gland enlargement } \\
\hline Chronic ( $\geq 12$ months), $n$ (\%) & $3 / 13(23.1 \%)$ & $2 / 13(15.4 \%)$ \\
\hline Episodically persistent (2-12 months), $n(\%)$ & $1 / 13(7.7 \%)$ & 0 \\
\hline $\begin{array}{l}\text { Duration of parotid and/or submandibular swelling at the time of } \\
\text { biopsy, months, median (range) }\end{array}$ & $13(5-64)$ & $15(5-54)$ \\
\hline Anti-Ro/SSA and/or anti-La/SSB positive, $n$ (\%) & $\begin{array}{l}10 / 13 \\
(76.9 \%)\end{array}$ & $\begin{array}{l}11 / 13 \\
(84.6 \%)\end{array}$ \\
\hline Lymphadenopathy, $n$ (\%) & $5 / 13(38.5 \%)$ & $5 / 13(38.5 \%)$ \\
\hline Cryoglobulinemia, $n(\%)$ & $3 / 13(23.1 \%)$ & $3 / 13(23.1 \%)$ \\
\hline Cryoglobulinemic vasculitis, $n(\%)$ & $3 / 13(23.1 \%)$ & $2 / 13(15.4 \%)$ \\
\hline Serum monoclonal component, $n(\%)$ & $6 / 13(46.1 \%)$ & $5 / 13(38.5 \%)$ \\
\hline Rheumatoid factor positive, $n$ (\%) & $7 / 13(53.8 \%)$ & $\begin{array}{l}11 / 13 \\
(84.6 \%)\end{array}$ \\
\hline Leukopenia $\left(\mathrm{WBC}<4000 / \mathrm{mm}^{3}\right), n(\%)$ & $4 / 13(30.7 \%)$ & $5 / 13(38.5 \%)$ \\
\hline Low C4, n (\%) & $5 / 13(38.5 \%)$ & $5 / 13(38.5 \%)$ \\
\hline $\begin{array}{l}\text { *Patients with both parotid and submandibular gland enlargemer } \\
\text { guided core needle biopsy. }\end{array}$ & guided CN & asound- \\
\hline
\end{tabular}

\section{Open biopsy controls}


Thirteen controls who underwent open surgical biopsy of the parotid gland were evaluated. Among them, $12 / 13(92.3 \%)$ could be classified as pSS (18), 10/13 (76.9\%) were females, the mean age was $51.8 \pm$ 15.2 years at the time of biopsy, and mean disease duration was $8.6 \pm 8.5$ years. All had parotid gland enlargement and 2/13 (15.4\%) had a concomitant submandibular gland enlargement (Table 1; Additional Table S1).

\section{Sonographic Features Of The Glands Subjected To US- guided CNB}

The inhouse-performed ultrasound evaluation was conducted in all 13 cases who underwent US-guided CNB. In 10/13 (76.9\%) patients, a focal sonographically suspicious lesion was identified and biopsied (Fig. 2). In 3/13 (23.1\%) the glandular parenchyma was diffusely inhomogeneous with no focal lesion.

In $9 / 13(69.2 \%)$ cases the biopsy was performed on the parotid and in 4/13 (30.8\%) on the submandibular gland. A minimum of 2 cores were taken in cases without a focal lesions. In cases with a sonographically suspicious focal lesion, a maximum of 4 cores were taken from the lesion and 1 in the surrounding parenchyma. The median (range) number of CNBs per gland per procedure was $4(2-5)$.

In 5/10 (50\%) cases, the most suspicious lesion was as a hypoechoic nodule with sonographic features of a hypertrophic lymphoid structure (range of diameters between $10 \times 10 \mathrm{~mm}$ and $23 \times 7 \mathrm{~mm}$ ). Three out of these five (60\%), displayed lobulated margins (Fig. 2). In 5/10 (50\%) cases, the most suspicious lesion was a limited hypoechoic area which was larger and/or more confluent than the remaining parenchyma (Fig. 2; Fig. 4).

\section{Histopathological Diagnoses}

\section{US-guided CNB cases}

In all cases of US-guided CNB sufficient high quality tissue was retrieved for pathologic diagnosis. The amount of tissue sampled ranged from 9.70 to $46.51 \mathrm{~mm}^{2}$ with a mean of $19.21 \mathrm{~mm}^{2}$ which was sufficient to perform also immunohistochemical $(n=13)$ and molecular analyses $(n=7)$.

The pathological diagnoses were: MZL-MALT in 5/13 (38.4\%), LymphoEpithelial SialAdenitis (LESA) in 1/13 (7.7\%), diffuse lymphocytic sialadenitis lacking LELs in 1/13 (7.7\%), 3/13 (23.1\%) other specific entities (one granulomatous sialadenitis consistent with sarcoidosis, one lgG4-related disease and one chronic sclerosing sialadenitis) (Fig. 3$)(3,4,26,27)$. Other miscellaneous entities were identified in $3 / 13$ (23.1\%) patients (Table 2). 
Table 2

Pathological diagnoses of patients submitted to ultrasound-guided core needle biopsy (cases).

\section{Cases with focal lesions \\ Diagnosis of the lesions}

\begin{tabular}{lll} 
Case \#1 & MZL-MALT Iymphoma & MZL-MALT lymphoma \\
Case \#2 & MZL-MALT lymphoma & MZL-MALT lymphoma \\
Case \#3 & Reactive lymph-node & $\begin{array}{l}\text { Focal chronic inflammatory } \\
\text { infiltrate* }\end{array}$ \\
\hline Case \#5 & LESA & LESA \\
\hline Case \#6 & Ductal cystic dilatation & $\begin{array}{l}\text { Focal chronic inflammatory } \\
\text { infiltrate* }\end{array}$ \\
\hline Case \#7 & Focal chronic inflammatory infiltrate* & Fibro-adipose tissue \\
\hline Case \#9 & MZL-MALT lymphoma & Diffuse chronic sialadenitis \\
\hline Case \#10 & IgG4-related disease & Diffuse chronic sialadenitis \\
\hline Case \#12 & MZL-MALT lymphoma & Fibro-adipose tissue \\
\hline Case \#13 & MZL-MALT lymphoma & MZL-MALT lymphoma \\
\hline $\begin{array}{l}\text { Cases without focal } \\
\text { lesions }\end{array}$ & Diagnosis & \\
\hline
\end{tabular}

\section{Adjacent parenchyma}

MZL-MALT Iymphoma

MZL-MALT Iymphoma

Reactive lymph-node

Granulomatous sialadenitis consistent with sarcoidosis

\section{Case \#8}

Diffuse chronic sialadenitis ${ }^{\circ}$

Case \#11

Chronic sclerosing sialadenitis

MZL-MALT: marginal zone lymphoma of the Mucosa-Associated Lymphoid Tissue Type; LESA: lymphoepithelial sialadenitis; *focal chronic inflammatory infiltrate, with a patchy lymphocytic infiltrate that could not be defined as sialadenitis; ${ }^{\circ}$ diffuse chronic sialadenitis, with a diffuse lymphocytic infiltrate that could be defined as sialadenitis and with no lymphoepithelial lesions.

All 5 cases of MZL-MALT showed a clonally restricted B-cell population by molecular tests.

When comparing the results of the biopsies performed within the sonographic lesions and the surrounding parenchyma, the diagnoses were the same in 3 MZL-MALT and in the LESA case. In one case of MZL-MALT, fibro-adipose tissue was found at the edge, and in another case, a diffuse chronic lymphocytic sialadenitis was observed (Table 2).

One of the cases merits a comment. Patient \#10 with a final diagnosis of IgG4-related disease fulfilled both the ACR-EULAR classification criteria for pSS (Additional Table S1) (18) and the ACR-EULAR IgG4related disease classification criteria (i.e. the histopathological evaluation of the submandibular gland 
displayed: dense lymphocytic infiltrate and storiform fibrosis with a $\lg G 4+: \lg G+$ ratio of $30 \%$ and the number of IgG4 + cells/hpf was between 10 and 50; serum IgG4 was 7x upper limit of normal) (26).

\section{Open biopsy controls}

All controls underwent a parotid biopsy of the "safety area" not guided by imaging detected lesions during the procedure. The side of the biopsy corresponded to the clinically most swollen gland. The amount of tissue sampled ranged from 6.66 to $118.33 \mathrm{~mm}^{2}$ with a mean of $32.75 \mathrm{~mm}^{2}$.

In 12/13 (92.3\%) controls open biopsy was effective in providing sufficient tissue for pathological diagnosis. In one control, only fibro-adipose tissue was sampled.

The diagnoses were: MZL-MALT in 4/12 (33.3\%), LESA in 2/12 (16.6\%), uncertain lymphoproliferative processes (defined as poor evidence for confluence of centrocyte-like lymphocytes and either oligoclonal IgH rearrangement or non-amplifiable DNA) in 4/12 (33.3\%), adipose metaplasia of the gland in 1/12 $(8.3 \%)$, and a mixed lymphocytic inflammation with granuloma in $1 / 12(8.3 \%)(3,4)$. The latter patient was submitted to 2 biopsies. Both biopsies did not contain salivary gland tissue, however, a mixed population of lymphoid cells, plasma cells and histiocytes was found forming granulomas partly centered by necrotic material, negative for Mycobacterium Tuberculosis by molecular test. Finally, a diagnosis of MZL-MALT was made based on the analysis of a retro-auricular lymph node sent together with the parotid samples.

\section{Sonographic Features Of Lymphoma US-guided CNB Cases}

All 5 cases that were diagnosed as MZL-MALT by histopathology were also suspicious for lymphoma based on their sonographic examination. In 2/5 (40\%) a hypoechoic nodule with hypertrophic lymphoid structure and lobulated margins was seen, and in 3/5 (60\%) a limited area with large and confluent hypoechoic lesions was detected (Fig. 2).

\section{Safety}

\section{US-guided CNB cases}

The procedure was well tolerated, with no serious and/or persistent complications occurring. A long-term complication was never observed. Transient complications were noticed in 6/13 (46.1\%) cases: 1 case of local swelling at the biopsy site lasting 2 days and 5 cases of mild local pain that resolved within 7 days without the need of analgesics (Table 3). 
Table 3

Complications of biopsy in cases and controls.

\begin{tabular}{|lll|}
\hline & US-guided CNB cases & Open biopsy controls \\
\hline Patients with complications, $n$ (\%) & $6 / 13(46.1 \%)$ & $12 / 13(92.3 \%)$ \\
\hline Transient complications, $n$ & 6 & 14 \\
\hline Persistent complications, $n$ & 0 & 2 \\
\hline Swelling at biopsy site, $n$ & US-guided CNB cases & Open biopsy controls \\
Haematoma, $n$ & 1 & 6 \\
\hline Bleeding, $n$ & 0 & 0 \\
\hline Local pain, $n$ & 0 & 0 \\
Local infection, $n$ & 5 & 8 \\
\hline Anaesthesia/paraesthesia, $n$ & 0 & 0 \\
Sialocele or fistula & 0 & 2 \\
\hline US-guided CNB: ultrasound-guided core needle biopsy; transient complications: $<12$ weeks; persistent \\
complications $\geq 12$ weeks.
\end{tabular}

\section{Open biopsy controls}

The procedure was well tolerated, although two patients had persistent complications (mild paresthesia of the ear lobe still present 8 months after the biopsy, and 1 case of mild facial anesthesia resolved in six months) were observed. Transient complications were reported in 12/13 (92.3\%) controls, overlapping in some of them: 6 cases of local swelling at the biopsy site lasting for a maximum of 7 days, and 8 cases of mild local pain that resolved within 7 days without the need of analgesics (Table 3).

\section{Discussion}

In this study, US-guided CNB of the SGs proved to be accurate and safe in pSS patients with persistent SGs enlargement, since it provided sufficient material for histopathological diagnosis without serious or permanent complications. Furthermore, US-guided CNB allowed the sampling of glandular areas with different sonographic patterns both in the parotid and submandibular glands.

A major issue of US-guided CNB is represented by the adequacy of the material obtained for histopathological evaluation and, if needed, for immunohistochemical and molecular tests, thus allowing a complete characterization of a possible lymphoproliferative disease $(11,12)$. 
As a matter of fact, given the number of cores obtained for each patient, ranging from 2 to 5 , the material was always sufficient to carry out standard immunohistochemistry in the suspicion of lymphoma and to characterize unexpected lesions (granulomatous sialadenitis, IgG4-related disease and chronic sclerosing sialadenitis); furthermore, it was also sufficient for molecular tests to evaluate B cell clonality.

Other two factors influence the adequacy of the material obtained, and both are operator-dependent, i.e., the number of cores performed that is based on his/her experience in evaluating SGs lesions and the choice of the gauge of the needle that, in this series, always allowed to characterize lesions even with two cores $(11,12)$.

In controls, open surgical parotid biopsies allowed the sampling of adequate material for pathological evaluation, however, a rather restricted glandular region ("safety zone") is biopsied that may not be the one showing the fully-fledged features of lymphoma. This non targeted approach may in part justify the relatively high number of biopsies diagnosed as "uncertain lymphoproliferative lesion" on the basis of morphology. On the contrary, with US-guided CNB, areas with different US patterns may be sampled allowing to target the biopsy to the sonographic focal lesion most suspicious for lymphoma thus reducing the possibility of false-negative results.

When dealing with the pathologic results in different biopsies from both the sonographic focal lesions and from the surrounding parenchyma, the biopsies were "discordant" in sixty percent of cases. Importantly, one pSS case with a final diagnosis of MALT lymphoma had such pathological finding only in the biopsy targeted to the most suspicious focal lesion and not in the biopsy from the surrounding parenchyma, supporting the notion that different sonographic features may be frequently associated with different pathologic patterns. The comparison of two different glandular areas could be useful to better evaluate the whole and still unsolved issues of the pathogenesis, diagnosis and treatment response in pSS $(28,29)$.

US-guided CNB has two further advantages. The first is the one of an easier access to the unexplored areas of the parotid gland as well as to the submandibular gland without having to resort to taxing surgery. In particular, there are only few reports of submandibular US-guided CNB in pSS patients (30). In this series, US-guided CNB of the submandibular glands proved to be safe and effective in providing sufficient material for diagnosis. The second advantage of US-guided CNB is that it allows to evaluate unexpected lesions in major SGs of pSS patients (granulomatous sialadenitis consistent with sarcoidosis, IgG4-related disease and chronic sclerosing sialadenitis) supporting the importance of SGs biopsy in pSS patients with glandular enlargement also for the detection of less frequent diseases that would otherwise be under-diagnosed and to understand their eventual relation to pSS $(31,32)$.

In our opinion, one key advantage of US-guided CNB in pSS is its overall safety, and feasibility. Indeed, it is a less invasive, less expensive and more patient-friendly technique than open surgical biopsy. In comparison to open surgical biopsy, we reported fewer long-term and transient complications, which have a high impact on the patient's acceptance to undergo biopsy. 
The main limitation of this study includes the small number of patients evaluated, although, it is the sole prospective cohort reported for US-guided CNB and compared to open biopsy in pSS patients with clinical suspicious of lymphoma.

\section{Conclusions}

In conclusion, US-guided CNB represents a novel, clinically relevant approach for the management of pSS patients with SGs enlargement with possible future research implications for tissue sampling (33).

\section{Abbreviations}

ACR-EULAR

American College of Rheumatology/ European League Against Rheumatism

ANA

antinuclear antibodies

LELS

lymphoepithelial lesions

LESA

LymphoEpithelial SialAdenitis

SG

salivary gland

MALT

Mucosa-Associated Lymphoid Tissue

MZL-MALT

Marginal Zone Lymphoma-Mucosa-Associated Lymphoid Tissue Type

pSS

primary Sjögren's syndrome

US-guided CNB

ultrasound-guided core needle biopsy

\section{Declarations}

\section{Ethics approval and consent to participate}

Written informed consent was obtained from each patients in accordance with the Declaration of Helsinki and with local guidelines for good clinical practice. The study was conducted according to a protocol approved by the Regional Ethical Committee (CEUR-2017-Os-027-ASUIUD).

\section{Consent for publication}

Informed consent was obtained from all participants for publication. 
Availability of data and materials

The dataset used and/or analysed during the currently study are available from the corresponding author on reasonable request.

\section{Competing interests}

The authors declare that they have no competing interests.

\section{Funding}

No specific funding was received from any bodies in the public, commercial or not-for-profit sectors to carry out the work described in this article.

\section{Author's contributions}

AZ, SZC, SDV contributed to study conception and design.

$A Z, S Z C, M L, E P, C A S, S D V$ contributed to analysis and interpretation of data.

All authors contributed to acquisition of data and to draft the article or revise it critically for important intellectual content.

All authors agreed for all aspects of the work in ensuring that questions related to the accuracy or integrity of any part of the work are appropriately investigated and resolved.

All authors final approved of the version of the article to be published.

\section{Acknowledgements}

Not applicable.

\section{References}

1. Goules AV, Tzioufas AG. Primary Sjögren's syndrome: clinical phenotypes, outcome and the development of biomarkers. Immunol Res. 2017;65(1):331-44.

2. Nocturne G, Pontarini E, Bombardieri M, Mariette X. Lymphomas complicating primary Sjögren's syndrome: from autoimmunity to lymphoma. Rheumatology (Oxford). 2019 Mar 5.

3. Nakamura S, Ponzoni M. Marginal zone B-cell lymphoma: lessons from Western and Eastern diagnostic approaches. Pathology. 2020 Jan;52(1):15-29.

4. Swerdlow SH, Campo E, Harris NL, Jaffe ES, Pileri SA, Stein H, Thiele J Chapter Extranodal marginal zone lymphoma of mucosa. InWHO Classification of Tumours of Haematopoietic and Lymphoid Tissues. WHO Classification of Tumours, Revised 4th Edition, Volume 2., Cook JR, Isaacson PG, Chott A, Nakamura S, Muller-Hermelink HK, Harris NL. Swerdlow SH, pp259-262. 
5. De Vita S, Gandolfo S, Zandonella Callegher S, Zabotti A, Quartuccio L. The evaluation of disease activity in Sjögren's syndrome based on the degree of MALT involvement: glandular swelling and cryoglobulinaemia compared to ESSDAl in a cohort study. Clin Exp Rheumatol. 2018 Jun;36(3):1506. Suppl 112(.

6. Alunno A, Leone MC, Giacomelli R, Gerli R, Carubbi F. Lymphoma and Lymphomagenesis in Primary Sjögren's Syndrome. Front Med. 2018;5:102.

7. De Vita S, Gandolfo S. Predicting lymphoma development in patients with Sjögren's syndrome. Expert Rev Clin Immunol. 2019;15(9):929-38.

8. Delli K, Vissink A, Spijkervet FKL. Salivary gland biopsy for Sjögren's syndrome. Oral Maxillofac Surg Clin North Am. 2014 Feb;26(1):23-33.

9. Spijkervet FKL, Haacke E, Kroese FGM, Bootsma H, Vissink A. Parotid Gland Biopsy, the Alternative Way to Diagnose Sjögren Syndrome. Rheum Dis Clin North Am. 2016;42(3):485-99.

10. Colella G, Cannavale R, Vicidomini A, Itro A. Salivary gland biopsy: a comprehensive review of techniques and related complications. Rheumatology (Oxford). 2010 Nov;49(11):2117-21.

11. Kim HJ, Kim JS. Ultrasound-guided core needle biopsy in salivary glands: A meta-analysis. Laryngoscope. 2018;128(1):118-25.

12. Witt BL, Schmidt RL. Ultrasound-guided core needle biopsy of salivary gland lesions: a systematic review and meta-analysis. Laryngoscope. 2014 Mar;124(3):695-700.

13. De Vita S, Lorenzon G, Rossi G, Sabella M, Fossaluzza V. Salivary gland echography in primary and secondary Sjögren's syndrome. Clin Exp Rheumatol. 1992 Aug;10(4):351-6.

14. Zabotti A, Zandonella Callegher S, Gandolfo S, Valent F, Giovannini I, Cavallaro E, et al. Hyperechoic bands detected by salivary gland ultrasonography are related to salivary impairment in established Sjögren's syndrome. Clin Exp Rheumatol. 2019 Jun;37(3):146-52. Suppl 118(.

15. Devauchelle-Pensec V, Zabotti A, Carvajal-Alegria G, Filipovic N, Jousse-Joulin S, De Vita S. Salivary gland ultrasonography in primary Sjögren's syndrome: opportunities and challenges. Rheumatology (Oxford). 2019 Mar 19. pii:kev079.

16. Jousse-Joulin S, Milic V, Jonsson MV, Plagou A, Theander E, Luciano N, et al. Is salivary gland ultrasonography a useful tool in Sjögren's syndrome? A systematic review. Rheumatology. 2016 May;55(5):789-800.

17. Mossel E, Delli K, van Nimwegen JF, Stel AJ, Kroese FGM, Spijkervet FKL, et al. Ultrasonography of major salivary glands compared with parotid and labial gland biopsy and classification criteria in patients with clinically suspected primary Sjögren's syndrome. Ann Rheum Dis. 2017 Nov;76(11):1883-9.

18. Shiboski CH, Shiboski SC, Seror R, Criswell LA, Labetoulle M, Lietman TM, et al. 2016 American College of Rheumatology/European League Against Rheumatism Classification Criteria for Primary Sjögren's Syndrome: A Consensus and Data-Driven Methodology Involving Three International Patient Cohorts: ACR/EULAR CLASSIFICATION CRITERIA FOR PRIMARY SS. Arthritis \& Rheumatology. 2017 Jan;69(1):35-45. 
19. Retamozo S, Brito-Zerón P, Ramos-Casals M. Prognostic markers of lymphoma development in primary Sjögren syndrome. Lupus. 2019 Jul;28(8):923-36.

20. Quartuccio L, Isola M, Baldini C, Priori R, Bartoloni Bocci E, Carubbi F, et al. Biomarkers of lymphoma in Sjögren's syndrome and evaluation of the lymphoma risk in prelymphomatous conditions: results of a multicenter study. J Autoimmun. 2014 Jun;51:75-80.

21. HarmonicSS. - HARMONIzation and integrative analysis of regional, national and international Cohorts on primary Sjögren's Syndrome (pSS) towards improved stratification, treatment and health policy making. Available from: https://www.harmonicss.eu/.

22. Haugen BR, Alexander EK, Bible KC, Doherty GM, Mandel SJ, Nikiforov YE, et al. 2015 American Thyroid Association Management Guidelines for Adult Patients with Thyroid Nodules and Differentiated Thyroid Cancer: The American Thyroid Association Guidelines Task Force on Thyroid Nodules and Differentiated Thyroid Cancer. Thyroid. 2016 Jan;26(1):1-133.

23. Mitsias DI, Kapsogeorgou EK, Moutsopoulos HM. Sjögren's syndrome: why autoimmune epithelitis? Oral Dis. 2006;12(6):523-32.

24. Kroese FGM, Haacke EA, Bombardieri M. The role of salivary gland histopathology in primary Sjögren's syndrome: promises and pitfalls. Clin Exp Rheumatol. 2018 Jun;36(3):222-33. Suppl 112 (.

25. Carbone A, Gloghini A, Ferlito A. Pathological features of lymphoid proliferations of the salivary glands: lymphoepithelial sialadenitis versus low-grade B-cell lymphoma of the malt type. Ann Otol Rhinol Laryngol. 2000 Dec;109(12 Pt 1):1170-5.

26. Wallace ZS, Naden RP, Chari S, Choi HK, Della-Torre E, Dicaire J-F, et al. The 2019 American College of Rheumatology/European League Against Rheumatism classification criteria for IgG4-related disease. Annals of the Rheumatic Diseases. 2020 Jan 1;79(1):77-87.

27. Peuraharju E, Saarinen R, Aro K, Mäkinen LK, Tarkkanen J, Mäkitie A, et al. Sclerosing sialadenitis of the submandibular gland is rarely an immunoglobulin G4-related disease in the Finnish population. Mod Pathol. 2020;33(4):551-9.

28. De Vita S, De Marchi G, Sacco S, Gremese E, Fabris M, Ferraccioli G. Preliminary classification of nonmalignant B cell proliferation in Sjögren's syndrome: perspectives on pathobiology and treatment based on an integrated clinico-pathologic and molecular study approach. Blood Cells Mol Dis. 2001 Aug;27(4):757-66.

29. De Vita S, Boiocchi M, Sorrentino D, Carbone A, Avellini C, Dolcetti R, et al. Characterization of prelymphomatous stages of B cell lymphoproliferation in Sjögren's syndrome. Arthr Rhuem. 1997;40(2):318-31.

30. Baer AN, Grader-Beck T, Antiochos B, Birnbaum J, Fradin JM. Ultrasound-guided biopsy of suspected salivary gland lymphoma in Sjögren's syndrome. Arthritis Care \& Research (Hoboken). 2020 Apr 5.

31. Fragoulis G, Zampeli E, Moutsopoulos H. IgG4-related sialadenitis and Sjögren's syndrome. Oral Dis. 2017 Mar;23(2):152-6.

32. Hong X, Li W, Xie X-Y, Zhang Z-Y, Chen Y, Gao Y, et al. Differential diagnosis of IgG4-related sialadenitis, primary Sjögren syndrome, and chronic obstructive submandibular sialadenitis. $\mathrm{Br} \mathrm{J}$ 
Oral Maxillofac Surg. 2017 Feb;55(2):179-84.

33. Pijpe J, Kalk WWI, van der Wal JE, Vissink A, Kluin PhM, Roodenburg JLN, et al. Parotid gland biopsy compared with labial biopsy in the diagnosis of patients with primary Sjogren's syndrome. Rheumatology. 2006 Jul 28;46(2):335-41.

\section{Figures}

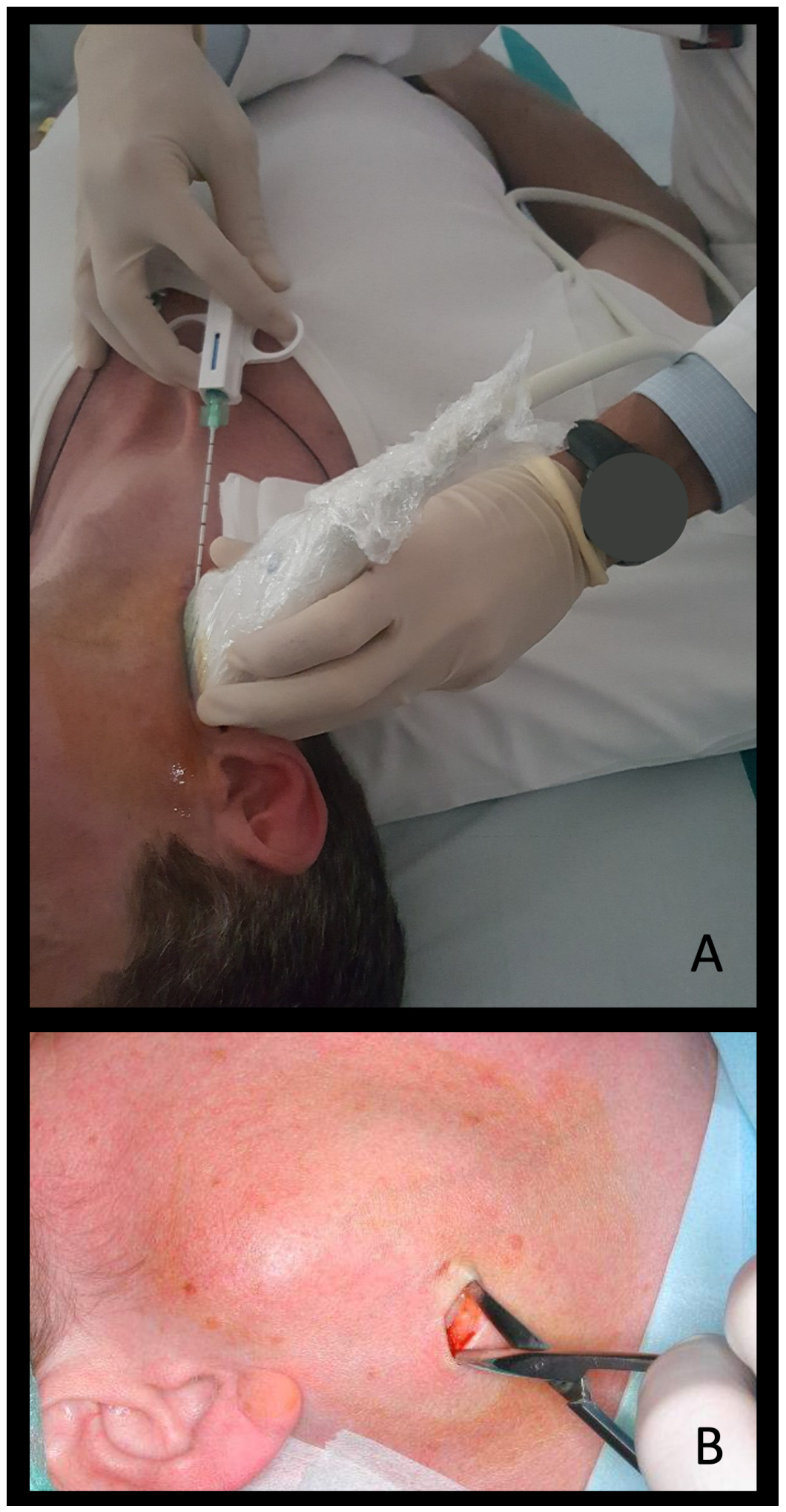




\section{Figure 1}

A) Ultrasound-guided core needle biopsy of the parotid gland. Biopsy was performed with a 14-gauge semi-automatic core biopsy needle system. The patient was in supine position with hyperextension of the neck and the head slightly turned towards the side opposite to that being biopsied. B) Open surgical parotid biopsy performed in the "safety zone", one centimetre beneath the ear lobe in order to avoid the main trunk of the facial nerve.

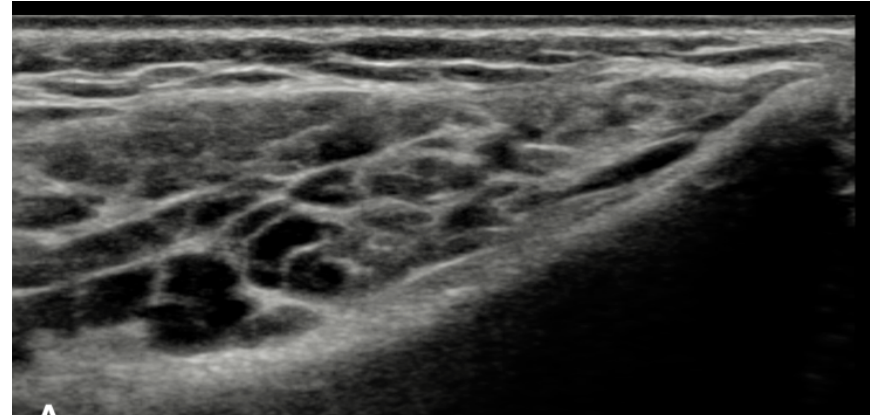

A

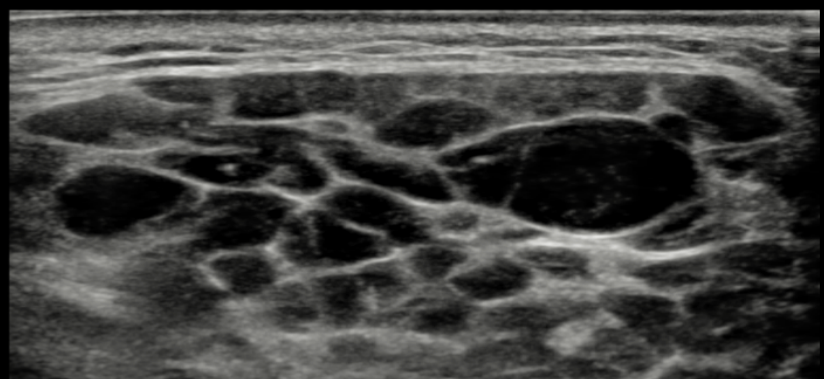

B
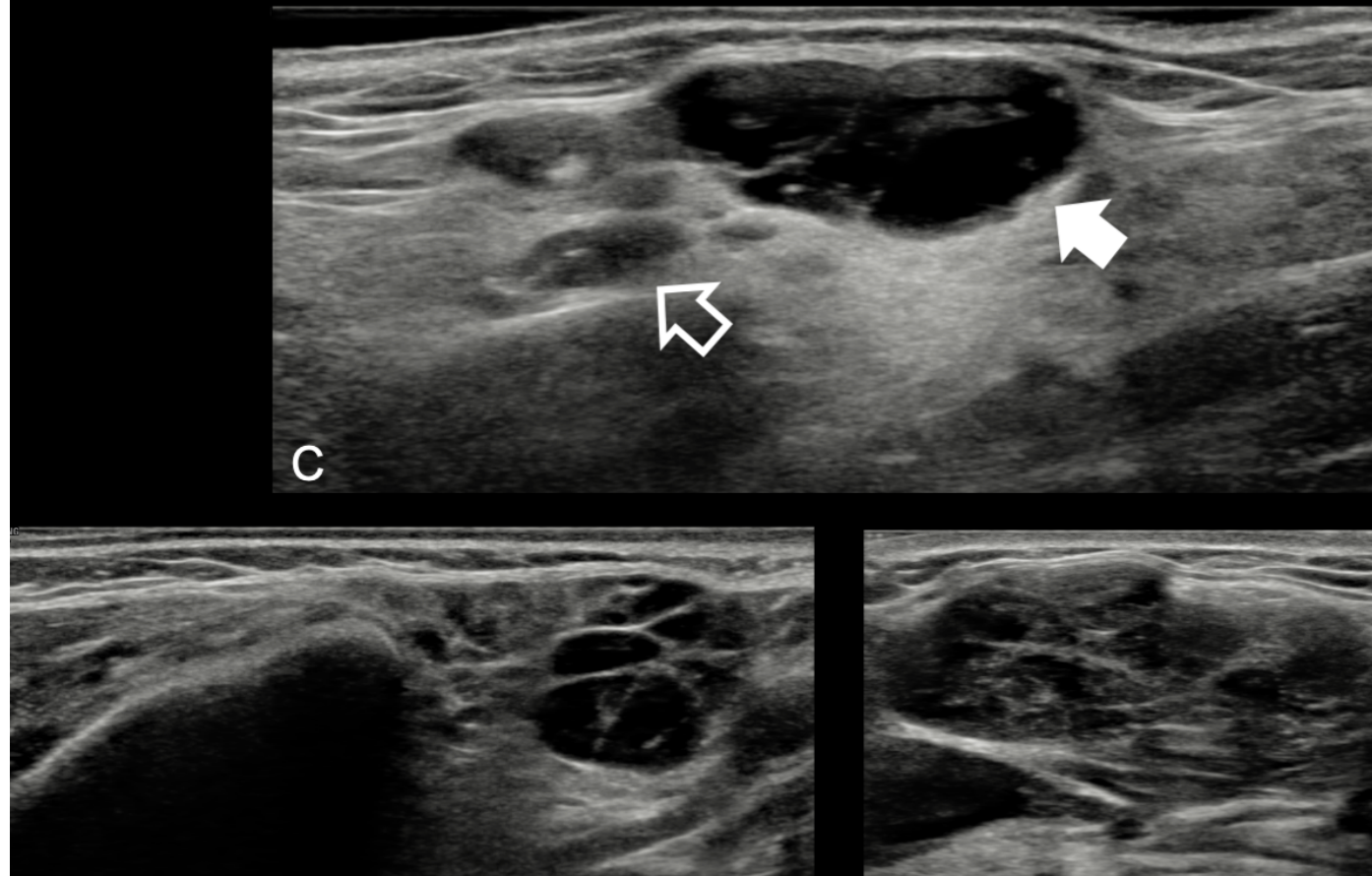

D
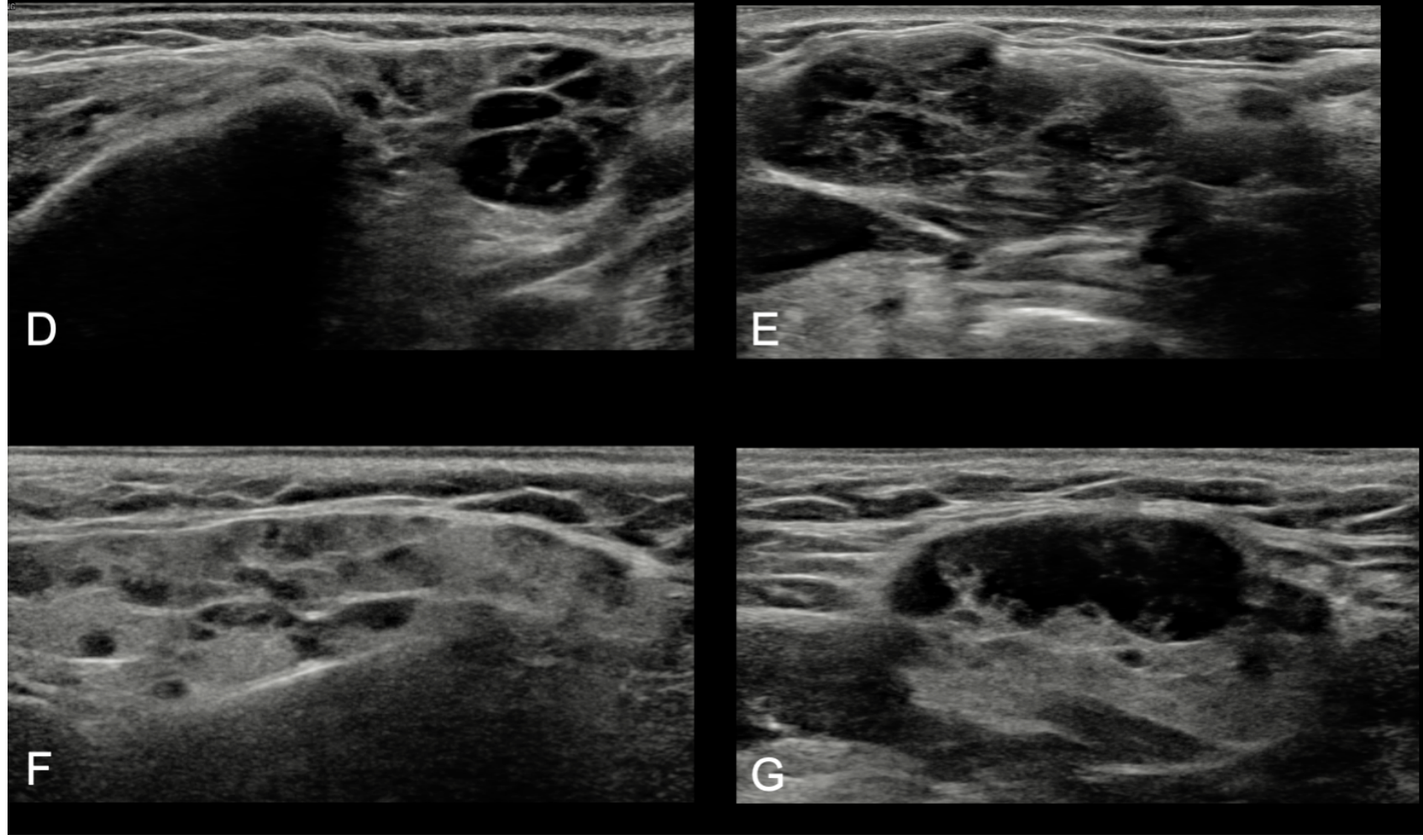


\section{Figure 2}

Ultrasound images of significant cases. A) Case \#2 and B) Case \#13, cases of marginal zone lymphoma of the Mucosa-Associated Lymphoid Tissue Type (MZL-MALT) of the parotid gland. C) Case\#9, the biopsy of the focal suspicious glandular lesion (white arrow) reported a diagnosis of MZL-MALT lymphoma of the parotid gland, while the biopsy of the surrounding parenchyma (void arrow) reported a diagnosis of diffuse chronic sialadenitis. D) Case \#5case, LymphoEpithelial SialAdenitis of the parotid gland. E) Case \#11, chronic sclerosing sialadenitis of the submandibular gland. F) Case \#4, granulomatous sialadenitis consistent with sarcoidosis of the parotid gland. G) Case \#10, IgG4-related diseases of the submandibular gland
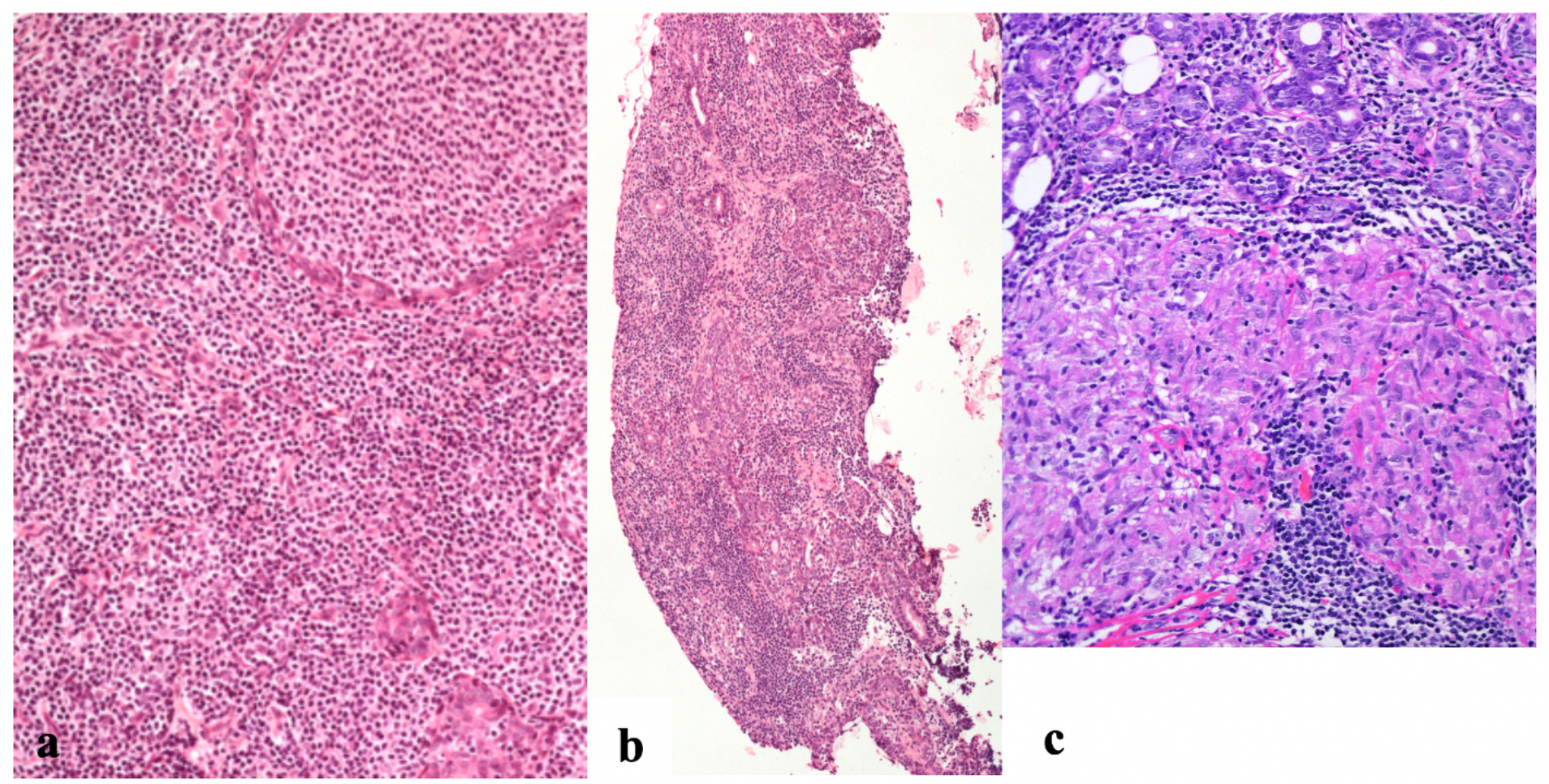

\section{Figure 3}

Histopathological images of significant cases. a) Case \#2, marginal zone lymphoma of the MucosaAssociated Lymphoid Tissue Type (MZL-MALT) showing a diffuse coalescence of centrocyte-like lymphocytes (H\&E, 20x). b) Case \#5, lympho-epithelial sialadenitis showing a dense infiltrate and, on the right, at the border, a lympho-epithelial lesion (H\&E, 4x). c) Case \#4, granulomatous sialadenitis consistent with sarcoidosis, showing non confluent granulomas lacking a necrotic core, with no multinucleated giant cells (PAS stain, 40x). 

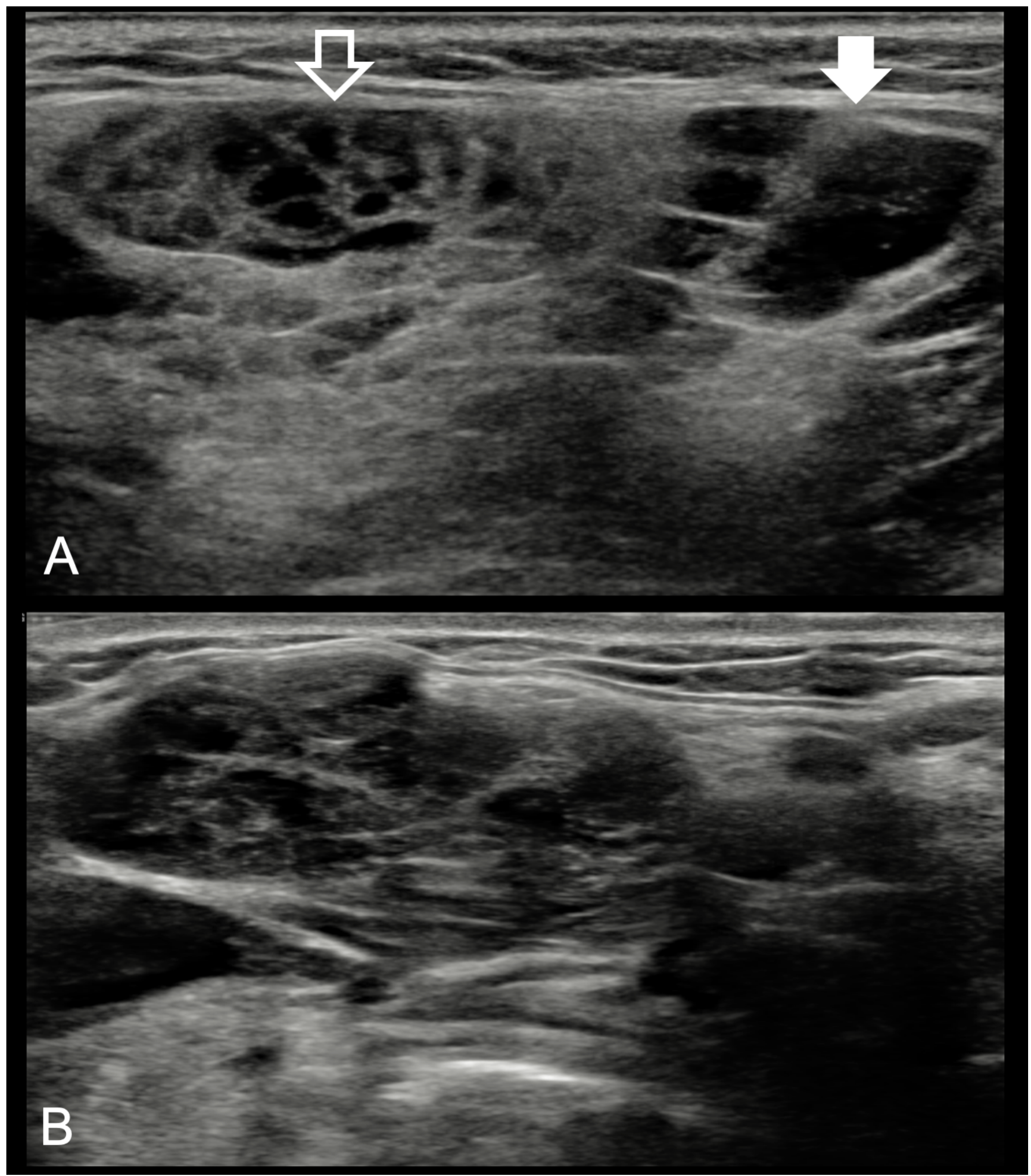

\section{Figure 4}

Ultrasound images. A) A case of marginal zone lymphoma of the Mucosa-Associated Lymphoid Tissue Type (MZL-MALT) of the parotid gland. White arrow: focal suspicious glandular lesion: a limited area within the parotid with hypoechoic lesions larger and/or more confluent than in the remaining parenchyma. Void arrow: inhomogeneity of the parenchyma typical for pSS. B) Diffuse inhomogeneity of the submandibular gland without focal glandular lesion. 


\section{Supplementary Files}

This is a list of supplementary files associated with this preprint. Click to download.

- Supplementalmaterial.docx 